\title{
LES flamelet-progress variable modelling and measurements of a turbulent partially-premixed dimethyl ether jet flame
}

\author{
S. Popp ${ }^{\mathrm{a}, *}$, F. Hunger ${ }^{\mathrm{a}}$, S. Hartl ${ }^{\mathrm{a}}$, D. Messiga ${ }^{\mathrm{a}}$, B. Coriton ${ }^{\mathrm{b}}$, J. H. Frank ${ }^{\mathrm{b}}$, F. \\ Fuest $^{\mathrm{c}}$, C. Hasse ${ }^{\mathrm{a}}$ \\ ${ }^{a}$ Chair of Numerical Thermo-Fluid Dynamics, ZIK Virtuhcon, Technische Universität \\ Bergakademie Freiberg, Freiberg, Germany \\ ${ }^{b}$ Combustion Research Facility, Sandia National Laboratories, Livermore, CA, USA \\ ${ }^{c}$ Department of Mechanical and Aerospace Engineering, The Ohio State University, \\ Columbus, OH, USA
}

\begin{abstract}
In the present study the flame structure of a piloted partially-premixed dimethyl ether flame (DME-D), which is based on the Sydney/Sandia piloted jet burner flame series, is investigated using an LES-flamelet-progress variable approach (LES-FPV). Simulation results are used together with a comprehensive experimental data set including multi-scalar measurements of temperature and major species from Raman/Rayleigh scattering, intermediate species $\mathrm{CH}_{2} \mathrm{O}$ and $\mathrm{OH}$ from laser induced fluorescence (LIF) and velocity data from particle image velocimetry (PIV). The comparison between nu-
\end{abstract}

\footnotetext{
* Corresponding author

TU Bergakademie Freiberg

Chair of Numerical Thermo-Fluid Dynamics

Fuchsmühlenweg 9

09599 Freiberg

Germany

Phone: +49 3731/ 39 4823, Fax: +49 3731394555

Email address: sebastian.popp@iec.tu-freiberg.de (S. Popp)
} 
merical and experimental data includes the mean and the root mean square (RMS) radial profiles for velocity, mixture fraction, temperature and mole fractions of major species at different downstream locations. Furthermore, species distributions conditioned on the experimentally accessible mixture fraction are compared and differences between DME and methane flames are discussed. In addition to this comparison, the computation of $\mathrm{CH}_{2} \mathrm{O}$ LIF and OH-LIF signals as well as the effective Rayleigh cross-section was incorporated into the flamelet-progress variable approach. The filtered and time-averaged numerical results are then directly compared with the corresponding experimental signals at different axial positions. The characteristic separation of instantaneous $\mathrm{CH}_{2} \mathrm{O}$ and $\mathrm{OH}$ fields, which was previously observed in simultaneous LIF measurements, is discussed and analyzed based on the underlying flamelet structures. Finally, modeling assumptions from the experimental post-processing for the effective Rayleigh cross-section, which were introduced to account for the experimentally inaccessible intermediate hydrocarbons, are evaluated using the detailed species composition from the numerical simulations.

Keywords: LES-FPV, DME, effective Rayleigh cross-section, $\mathrm{CH}_{2} \mathrm{O}-\mathrm{LIF}$, OH-LIF

\section{Introduction}

Dimethyl ether (DME) is a promising low-emission and environmentallyfriendly alternative fuel, especially for compression ignition engines [1]. While the majority of DME is currently produced from fossil fuels like coal and natural gas, there is potential that DME will be produced from biomass 
resources and could become an important bio-fuel [2]. DME also serves as a sensible starting point for systematic studies of turbulent combustion of more complex oxygenated fuels.

Investigations into more complex fuels compared to methane, such as DME, for use in practical applications require a thorough understanding of turbulence-chemistry-interaction (TCI). Turbulent jet flames have become a paradigm for such investigations. In particular, the Sydney/Sandia piloted turbulent jet flames became a well-established benchmark case for investigations of turbulence-chemistry-interaction within the TNF workshop [3]. Several experimental studies for this flame series (flames C-F) were conducted [4 7] with $\mathrm{CH}_{4}$ as a fuel, focusing on turbulence-chemistry-interaction and flame structure. Furthermore, the detailed measurements provide a good basis for model validation. Various numerical approaches and combustion models, such as transported PDF methods [8 11], conditional moment closure (CMC) [12 -15] and flamelet approaches [16-20] were used to model these turbulent jet flames.

Recently, some initial studies using the chemically more complex fuel DME were performed within this context. Experimental investigations by Gabet et al. [21] of the turbulent flames DLR A and B showed significant differences in the flame structure for DME compared to $\mathrm{CH}_{4}$. Further studies considered a series of partially-premixed DME/air flames in the Sydney/Sandia piloted jet burner [22]. Coriton et al. 23] analysed the topology of $\mathrm{CH}_{2} \mathrm{O}$ and $\mathrm{OH}$ fields using laser-induced fluorescence (LIF). Additionally, radial velocity distributions at different axial locations were obtained using stereoscopic particle image velocimetry (SPIV). Initial computational results 
using LES-CMC for the velocity, $\mathrm{CH}_{2} \mathrm{O}$ and $\mathrm{OH}$ fields were also presented in [23]. This investigation showed characteristic separations of $\mathrm{CH}_{2} \mathrm{O}$ and $\mathrm{OH}$ distributions in DME flames. Further, it was shown that the influence of the chemical mechanism is relevant for the absolute $\mathrm{CH}_{2} \mathrm{O}$ values in the context of LES-CMC. Fuest et al. 24] investigated the flame structure of the flames DME-D and DME-F using one-dimensional Raman/Rayleigh/CO-LIF measurements for the temperature and major species $\left(\mathrm{CO}, \mathrm{CO}_{2}, \mathrm{H}_{2}, \mathrm{H}_{2} \mathrm{O}\right.$, $\mathrm{O}_{2}, \mathrm{~N}_{2}, \mathrm{DME}$ ). The comparison with the corresponding piloted $\mathrm{CH}_{4}$ flames showed that extinction events occur more rarely when DME is used as a fuel. Differences in the flame structure of $\mathrm{CH}_{4}$ and DME jet flames were attributed to differences in fuel consumption and product species formation in the fuel-rich region.

The reported differences between methane and DME flames, and the availability of comprehensive experimental data are the motivation for this work. Here, the scope is the investigation of the turbulent flame structure of the DME-D flame using a flamelet-progress variable approach in an LES framework (LES-FPV). In particular, there is an analysis of the characteristic features of the chemically more complex DME flames, which exhibit fundamental differences compared with the well-known $\mathrm{CH}_{4}$ flames. The rapid DME pyrolysis leads to increased levels of formaldehyde $\left(\mathrm{CH}_{2} \mathrm{O}\right)$ in fuel-rich regions [21], levels which are two orders of magnitude larger than for $\mathrm{CH}_{4}$ flames. Besides higher amounts of $\mathrm{CH}_{2} \mathrm{O}$, the interplay with $\mathrm{OH}$ radicals showed varying separations in the distributions of the two species, which were detected by LIF measurements [23]. The experimental results by Coriton et al. [23] and Fuest et al. 24] provide a comprehensive data set including 
velocity, species and temperature data, which we use for comparison with the LES results.

In addition to this direct comparison, we also apply the approach proposed by Connelly et al. 25] to simulate the LIF and Rayleigh signals from the detailed numerical results. We compare statistics of simulated and measured signals for OH-LIF, $\mathrm{CH}_{2} \mathrm{O}-\mathrm{LIF}$, and Rayleigh scattering. This complementary approach to comparing simulations and measurements is well suited for combustion of complex fuels such as DME, since the evaluation of measured Raman and Rayleigh signals is more complicated, as discussed in [26]. The decomposition of DME in the fuel rich-region leads to the formation of significant amounts of intermediate hydrocarbons. Since these species contribute to the same Raman detection channel, species separation is almost impossible. The evaluation of Rayleigh measurements is also affected, since intermediate hydrocarbons exhibit significant differences in their specific cross-sections and the resulting effective Rayleigh cross-section. Therefore, we use the detailed local knowledge of the coupled species composition from the numerical simulations to evaluate potential improvements when post-processing Rayleigh signals.

The remainder of the paper is structured as follows. Section 2 describes the LES-FPV approach and presents the procedures for LIF and Rayleigh signal computations. In Section 3 the burner setup is described and the experimental approaches are briefly summarized. Furthermore, the numerical setup is described. Section 4 discusses the results and compares them with experimental data. The results are summarized in Section 5 . 


\section{Numerical methods}

In this study a coupled LES-FPV approach is used to predict turbulent combustion. The flow field is described by the Favre-filtered Navier-Stokes equations, including mass-, momentum- and total enthalpy transport. Closure is achieved by means of an eddy viscosity hypothesis and the subgrid turbulence, including the computation of the eddy viscosity $\mu_{\mathrm{t}}$, is evaluated with the sigma model [27], applying a fixed model constant, $C_{\sigma}=1.5$.

\subsection{Flamelet-progress variable approach}

The FPV approach requires an additional transport equation to be solved for the conserved scalar mixture fraction $\widetilde{Z}$. For the mixture fraction variance $\widetilde{Z^{\prime \prime 2}}$ an algebraic equation is used [28], where $\Delta$ is the filter width and $C_{\widetilde{Z^{\prime \prime 2}}}=$ 0.13 is the model constant. Furthermore, an equation is required for the nonnormalized progress variable $\widetilde{Y}_{C}$. In the present study, the progress variable is defined as the sum of the species mass fractions of $\mathrm{H}_{2}, \mathrm{H}_{2} \mathrm{O}, \mathrm{CO}$ and $\mathrm{CO}_{2}$,

$$
\begin{gathered}
\frac{\partial}{\partial t}(\bar{\rho} \widetilde{Z})+\nabla \cdot(\bar{\rho} \widetilde{\mathbf{u}} \widetilde{Z})=\nabla \cdot\left[\left(\bar{\rho} D_{Z}+\frac{\mu_{\mathrm{t}}}{\mathrm{Sc}_{\mathrm{t}}}\right) \nabla \widetilde{Z}\right] \\
\widetilde{Z^{\prime \prime 2}}=C_{\widetilde{Z^{\prime \prime 2}}} \Delta^{2}|\nabla \widetilde{Z}|^{2} \\
\frac{\partial}{\partial t}\left(\bar{\rho} \widetilde{Y}_{C}\right)+\nabla \cdot\left(\bar{\rho} \widetilde{\mathbf{u}} \widetilde{Y}_{C}\right)=\nabla \cdot\left[\left(\bar{\rho} D_{Y_{C}}+\frac{\mu_{\mathrm{t}}}{\mathrm{Sc}_{\mathrm{t}}}\right) \nabla \widetilde{Y}_{C}\right]+\overline{\dot{\omega}}_{Y_{C}} .
\end{gathered}
$$

Here, $D_{Z}$ and $D_{Y_{C}}$ denote the diffusion coefficient of the mixture fraction and progress variable, respectively. If unity Lewis numbers are assumed for all species, the two diffusion coefficients are equal. The turbulent Schmidt number $\mathrm{Sc}_{\mathrm{t}}$ is set to 0.4 . 
Radiation is described by an optically thin model [29]. The absorption coefficients of the radiative species $\mathrm{CH}_{4}, \mathrm{CO}, \mathrm{CO}_{2}$ and $\mathrm{H}_{2} \mathrm{O}$ are based on RADCAL simulations [30]. This approach is also suggested by the TNF [3].

The thermo-chemical state is retrieved from flamelet look-up tables (FLUTs). Both non-premixed and premixed flamelet configurations can be used to generate flamelet tables. For laminar non-premixed flamelets the corresponding equations for temperature and species mass fractions are solved in the mixture fraction space [31] for different scalar dissipation rates. Here we vary the scalar dissipation rate from zero up to a value close to the quenching limit, and more details are given below in section 4.3 . When differential diffusion effects are considered, this leads to more complex flamelet equations [32]. An in-house flamelet solver is used, which also performs the mapping from the scalar dissipation rate to the normalized progress variable. For the premixed flame configuration, flamelet tables are constructed from physical solutions of freely propagating premixed flames for different equivalence ratios [33].

A presumed $\beta$-shaped filtered density function (FDF) is used to integrate the mixture fraction, and a $\delta$-FDF is applied for the normalized progress variable to account for non-resolved fluctuations. Using a $\delta$-FDF means that no subgrid closure is employed for the normalized progress variable. For the integration process, the normalized progress variable $C$ is used to ensure statistical independence [34],

$$
C=\frac{Y_{C}-Y_{C, \text { min }}(Z)}{Y_{C, \text { max }}(Z)-Y_{C, \text { min }}(Z)} .
$$

Here, the minimum and the maximum progress variables are each a function of the mixture fraction. These values are obtained directly from the flamelet solutions. 


\subsection{Computation of LIF and Rayleigh signals}

In addition to the direct comparison of species mole fractions and temperature from Raman/Rayleigh measurements [24], we also compare calculations and measurements of $\mathrm{OH}-\mathrm{LIF}, \mathrm{CH}_{2} \mathrm{O}-\mathrm{LIF}$ and Rayleigh scattering signals following the approach used by Connelly et al. [25]. Below, the computation of the LIF and the Rayleigh scattering signals is described in detail. For the FPV approach, we computed the signals using the laminar (nonfiltered) flamelet solutions and then performed the FDF integration as for any other quantity. As a result, we obtained the filtered signals as a function of the mean mixture fraction, the mixture fraction variance and the progress variable. The filtered signals were stored in the flamelet look-up table and later retrieved in the LES calculation. This approach ensured that subgrid fluctuations were taken into account.

\subsection{1. $O H-L I F$}

The OH-LIF signals for the $\mathrm{Q}_{1}(6)$ transition of the $A^{2} \Sigma^{+} \leftarrow X^{2} \Pi^{+}$ $\left(v^{\prime}=1, v^{\prime \prime}=0\right)$ band were calculated using the following expression, which applies to the linear LIF regime with the excited-state decay rate dominated by collisional quenching,

$$
S_{\mathrm{OH}-\mathrm{LIF}} \propto N_{\mathrm{OH}} f_{J} \frac{A}{A+Q},
$$

where $N_{\mathrm{OH}}$ is the $\mathrm{OH}$ number density, $f_{J}$ is the Boltzmann fraction population of the absorbing state with rotational quantum number $J, A$ is the spontaneous emission rate, and $Q$ is the total collisional quenching rate of the excited state. The total quenching rate is the summation of the quenching rates for each of the collisional partners and is a function of the local 
temperature and composition given by

$$
Q=N_{\text {Tot }} \sum_{i} X_{i} \sigma_{i}\left(\frac{8 k_{\mathrm{B}} T}{\pi \mu_{i}}\right)^{0.5},
$$

where $N_{\text {Tot }}$ is the total number density, $k_{\mathrm{B}}$ is Boltzmann's constant, and $X_{i}$, $\sigma_{i}$, and $\mu_{i}$ are the mole fraction, quenching cross-section, and reduced mass, respectively, for quenching species $i$. Quenching cross-sections were obtained from Tamura et al. [35]. The downstream evolution of the computed and measured OH-LIF signals were compared after normalizing the signals by their corresponding reference values at $x / d=5$.

\subsection{2. $\mathrm{CH}_{2} \mathrm{O}-\mathrm{LIF}$}

In contrast to OH-LIF computations, the simulation of $\mathrm{CH}_{2} \mathrm{O}-\mathrm{LIF}$ signals is more complex, since it involves excitation of overlapping transitions and the temperature- and species-dependent quenching cross-sections are unknown. In this study, the same procedure as in [23] is used to compute the $\mathrm{CH}_{2} \mathrm{O}$-LIF signals. The LIF signal is described by Eqs. (5) and (6) with the addition of an overlap integral term that accounts for the spectral overlap of the laser lineshape with multiple $\mathrm{CH}_{2} \mathrm{O}$ absorption transitions. The temperature dependences of the Boltzmann fraction population and the overlap integral are calculated using spectral simulations and then are approximated through a temperature-dependent polynomial fit, which is used in the LES computations. The model for collisional quenching of $\mathrm{CH}_{2} \mathrm{O}-\mathrm{LIF}$ uses a temperature dependence of $T^{-0.5}$ for the quenching cross-sections of all species. As a result, the total quenching rate given by Eq. (6) is inversely proportional

to temperature. In the previous study of Coriton et al. [23], this quenching model provided slightly better agreement between LES calculations and mea- 
surements compared to a model using temperature-independent quenching cross-sections.

\subsubsection{Rayleigh signal and effective Rayleigh cross-section}

The Rayleigh signal $S_{\text {Ray }}$ is proportional to the number density $N$ and an effective Rayleigh cross-section

$$
S_{\text {Ray }}=C_{\text {exp }} I_{\text {laser }} N \sigma_{\text {Ray,eff }}
$$

The number density is inversely proportional to the temperature and the effective Rayleigh cross-section is determined by species mole fractions and their corresponding Rayleigh cross-sections $\sigma_{\text {Ray }, i}$

$$
\sigma_{\text {Ray,eff }}=\sum_{i} X_{i} \sigma_{\text {Ray }, i}
$$

We use the Rayleigh cross-sections reported by Fuest et al. [26], which are given in Tab. 11. Sixteen species $\left(\mathrm{CO}_{2}, \mathrm{CO}, \mathrm{O}_{2}, \mathrm{~N}_{2}, \mathrm{H}_{2}, \mathrm{H}_{2} \mathrm{O}, \mathrm{OH}, \mathrm{O}, \mathrm{H}\right.$, $\left.\mathrm{CH}_{4}, \mathrm{CH}_{3}, \mathrm{C}_{2} \mathrm{H}_{2}, \mathrm{C}_{2} \mathrm{H}_{4}, \mathrm{C}_{2} \mathrm{H}_{6}, \mathrm{CH}_{2} \mathrm{O}, \mathrm{DME}\right)$ are used to determine the effective Rayleigh cross-section and a mole weighted formulation for the effective Rayleigh cross-section, referred to as $\sigma_{\text {Ray,eff,ref, is applied. Along with species }}$ mole fractions, this quantity is calculated as the flamelet table is generated

$$
\sigma_{\text {Ray,eff,ref }}=\frac{\sum_{i} X_{i} \sigma_{\text {Ray }, i}}{\sum_{i} X_{i}}
$$

During the analysis of the experimental data in [24], assumptions were made to account for intermediate hydrocarbon mole fractions which were not accessible by the Raman measurements, due to spectral overlap on the Raman detection channel. In addition to DME, four intermediate hydrocarbons $\left(\mathrm{CH}_{4}, \mathrm{CH}_{3}, \mathrm{C}_{2} \mathrm{H}_{4}, \mathrm{C}_{2} \mathrm{H}_{6}\right)$ were considered for the Raman detection channel 
for hydrocarbons. Therefore, the mole fractions of these five species were combined into a hydrocarbon mole fraction, $X_{\mathrm{HC}}$. Each of these five species has different Rayleigh and Raman scattering cross-sections. As a result, the calculation of $X_{\mathrm{HC}}$ and temperature from the Raman and Rayleigh signals in rich regions requires a model to estimate the relative mole fractions of these hydrocarbons. A model, based on a laminar flame calculation, was used by Fuest et al. [26] to determine a temperature-dependent Rayleigh crosssection for all hydrocarbons $\sigma_{\text {Ray,HC }}$. The model-based effective Rayleigh cross-section is calculated by

$$
\sigma_{\text {Ray,eff,exp }}=\frac{\sum_{i=1}^{6} X_{i} \sigma_{\mathrm{Ray}, i}+X_{\mathrm{HC}} \sigma_{\mathrm{Ray}, \mathrm{HC}}}{\sum_{i=1}^{6} X_{i}+X_{\mathrm{HC}}},
$$

where the sum includes six major species $\left(\mathrm{CO}, \mathrm{CO}_{2}, \mathrm{H}_{2}, \mathrm{H}_{2} \mathrm{O}, \mathrm{O}_{2}, \mathrm{~N}_{2}\right)$. Based on the LES results in Appendix C an error estimation is presented to evaluate the impact of the temperature-dependent Rayleigh cross-section model $\sigma_{\text {Ray,HC }}$ in Eq. (10) on the fully resolved representation given in Eq. (9).

\section{Experimental and numerical setup}

In the following, the experimental and the numerical setup of the DMED flame is presented. First, the burner configuration is described. Then, in Sections 3.1 .2 and 3.1.3, the experimental approaches by Coriton et al. [23] and by Fuest et al. [24] are briefly summarized. In Section 3.2 the numerical setup for the LES is described. 


\subsection{Experimental setup}

\subsubsection{Burner configuration and boundary conditions}

The DME flame series is directly derived from the Sydney/Sandia flame series of piloted jet flames [3]. The fuel jet diameter is $d=7.45 \mathrm{~mm}$ and therefore slightly larger than the original diameter. The pilot annulus inner and outer diameters are $8 \mathrm{~mm}$ and $18.2 \mathrm{~mm}$, respectively. For the DME-D flame considered here the bulk velocity of the fuel jet is $u_{\text {bulk }}=45.9 \mathrm{~m} / \mathrm{s}$, which corresponds to a Reynolds number of $\mathrm{Re}=2930 \mathrm{~d}^{1}$. The unburned velocity of the pilot stream is $u_{\text {pilot }}=1.1 \mathrm{~m} / \mathrm{s}$ and the composition contains $\mathrm{C}_{2} \mathrm{H}_{2}, \mathrm{H}_{2}, \mathrm{CO}_{2}$ and $\mathrm{N}_{2}$, which were chosen to match a premixed DME flame with an equivalence ratio $\phi=0.6$. An air co-flow with a velocity of $u_{\text {co-flow }}=$ $0.9 \mathrm{~m} / \mathrm{s}$ surrounds the piloted burner. The unburned gas composition from the experiments [24] is given in Tab. 2.

\subsubsection{Experimental investigations by Coriton et al. [23]}

Coriton et al. [23] performed SPIV, OH-LIF and $\mathrm{CH}_{2} \mathrm{O}-\mathrm{LIF}$ measurements. For the velocity measurements a dual-headed Nd:YAG laser $(\lambda=$ $532 \mathrm{~nm}$ ) with a repetition rate of $5 \mathrm{~Hz}$ was used. Aluminium oxide tracer particles with a diameter of $0.3 \mu \mathrm{m}$ were seeded into the fuel jet and co-flow. The OH-LIF measurements were performed using a Nd:YAG pumped-dye laser $(\lambda=283.01 \mathrm{~nm})$, which was tuned to the $\mathrm{Q}_{1}(6)$ line of the $A^{2} \Sigma^{+} \leftarrow X^{2} \Pi^{+}$ $\left(v^{\prime}=1, v^{\prime \prime}=0\right) \mathrm{OH}$ band. For $\mathrm{CH}_{2} \mathrm{O}-\mathrm{LIF}$ an injection-seeded Nd:YAG laser was used. The laser was operated at $\lambda=354.83 \mathrm{~nm}$ to excite overlapping transitions in the $4_{0}^{1}$ band of the $\widetilde{A}^{1} A_{2} \leftarrow \widetilde{X}^{1} A_{2}$ system.

\footnotetext{
${ }^{1} \nu=1.167 \cdot 10^{-5} \mathrm{~m}^{2} / \mathrm{s}$
} 


\subsubsection{Experimental investigations by Fuest et al. 24.}

The experimental investigations by Fuest et al. 24] provided a set of multi-scalar data, including the temperature and major species $\left(\mathrm{CO}, \mathrm{CO}_{2}\right.$, $\left.\mathrm{H}_{2}, \mathrm{H}_{2} \mathrm{O}, \mathrm{O}_{2}, \mathrm{~N}_{2}, \mathrm{DME}\right)$. Instantaneous temperature and species concentrations were measured by simultaneous 1D Raman/Rayleigh scattering and CO-LIF. For spontaneous Raman/Rayleigh scattering, four sequentially-fired frequency-doubled Nd:YAG lasers $(\lambda=532 \mathrm{~nm})$ were used. Simultaneous CO-LIF was excited with a Nd:YAG-pumped tunable dye laser. The analysis of the flame structure was conducted using the ensemble mean and root mean square (RMS) radial profiles, and scatter plots, means and PDF's conditioned by the derived mixture fraction.

\subsection{Numerical setup}

The numerical investigations are conducted with a coupled LES-FPV solver based on OpenFOAM ${ }^{\circledR}$ 2.1. The simulations are performed on a stretched rectilinear mesh with a resolution of $200 \times 80 \times 80$ cells, using second-order discretization in time and space. The length of the computational domain is $50 \mathrm{~d}$ in the downstream direction and the size of the inlet

plane is $8 d \times 8 d$. To consider jet spreading, the mesh is stretched and the dimension of the outlet plane is $32 d \times 32 d$. Starting from the center of the jet inlet, a uniform mesh expansion is applied in each spatial direction. The lateral cell-to-cell expansion ratio is set to 1.03. In the downstream direction, this ratio is 1.01 . This leads to a resolution of $0.35 \mathrm{~mm}$ at the center of the jet inlet. The average resolution within the flame zone is $0.75 \mathrm{~mm}$ at $x / d=10$ and $1 \mathrm{~mm}$ at $x / d=20$.

Appropriate unsteady boundary conditions for the fuel jet are generated 
with an LES of the pipe flow. This data is used as instationary input for the velocity boundary condition. The pilot inflow velocity is adjusted based on the density ratio between burned and unburned composition and set to $u_{\text {pilot }}=5.8 \mathrm{~m} / \mathrm{s}$.

In two previous studies [23, 26], the chemical mechanisms proposed by Kaiser et al. [36] and Zhao et al. [37] were used. In the present study, we use the Zhao mechanism, which yields better agreement with published experimental data for the laminar burning velocity [26, 37]. These mechanisms also show notable differences in the predicted mole fractions of intermediate species, such as $\mathrm{CH}_{2} \mathrm{O}$ [23]. Based on a sensitivity analysis of different approaches for generating appropriate flamelet look-up tables (see Appendix A) a standard non-premixed flamelet approach with unity Lewis number is applied for the flame simulations, as reported in the main part of the paper. This is consistent with previous findings for the Sandia flame series, see e.g. [38].

\section{Results and Discussion}

In this section the LES-FPV results are compared to the experimental results gained by Coriton et al. [23] and Fuest et al. [24]. First, the velocity and the mixture fraction fields are analysed. Then there is a discussion of the radial temperature profiles and species distributions in physical and mixture fraction space. In the last two sections, 4.3 and 4.4, a comparison of the measured and computed LIF signals and Rayleigh scattering cross-section is shown. 


\subsection{Velocity and mixture fraction fields}

First, we look at the radial profiles of the mean and the RMS axial velocity shown in Fig. 11. The LES data was averaged in the azimuthal direction over 35 flow-through times ${ }^{2}$. The same procedure is used for all quantities presented below.

Overall, the results of the simulations and SPIV measurements are in good agreement. Near the jet inlet at $x / d=5$, the experimental mean profiles are slightly wider than the computational results. The agreement between the velocity profiles improves as the downstream position increases from $x / d=5$ to 20 . However, discrepancies are found for the RMS velocity, with the maximum fluctuations and the centerline values from the LES being slightly higher. Very similar trends in the downstream evolution of the computed velocity field were previously reported using an LES-CMC approach [23]. Note that comparisons of measurements and LES calculations are performed using Reynolds averaging of instantaneous velocities. However, the instantaneous LES realizations include a density-weighted contribution from the Favre-filtering that is inherent in the subgrid scale filter. Since the PIV measurements do not have a density weighting, some of the observed discrepancies might be related to this difference, especially in regions with a high contribution of modeled rather than resolved fluctuations e.g. close to the nozzle. Nevertheless, the comparisons indicate that the LES captures the overall structure of the velocity field sufficiently well for an appropriate prediction of mixture fraction transport.

\footnotetext{
${ }^{2}$ based on the bulk velocity of the jet, 1 flow-through time equals $8.1 \mathrm{~ms}$
} 
Next, we compare computed and measured radial profiles of mixture fraction. The mixture fraction from the Raman measurements is calculated from six major species $\left(\mathrm{CO}_{2}, \mathrm{CO}, \mathrm{O}_{2}, \mathrm{~N}_{2}, \mathrm{H}_{2}, \mathrm{H}_{2} \mathrm{O}\right)$ and five hydrocarbon species (DME, $\mathrm{CH}_{4}, \mathrm{CH}_{3}, \mathrm{C}_{2} \mathrm{H}_{4}, \mathrm{C}_{2} \mathrm{H}_{6}$ ). The definition for this adapted Bilger mixture fraction $Z=Z_{6+(5)}^{*}$ is directly taken from [26] and is stored in the lookup table as a function of the transported mixture fraction and the progress variable, see Eqs. (1)-(3). To be consistent with the experimental data this definition of the mixture fraction is used for the following comparison, instead of the transported mixture fraction.

The computed mean and RMS mixture fraction profiles between $x / d=5$ and $x / d=20$ generally agree well with the experimental data; this comparison is shown in Fig. 2, Differences primarily occur very close to the jet inlet $(x / d=1)$. The mean mixture fraction field of the simulation is slightly wider, at $x / d=1$. However, the downstream development of the computed profile is close to the measured one. The RMS values agree well along the radius except for an underestimation near the jet inlet. Since this formulation of the Bilger mixture fraction is directly computed from species solutions, the results depend on the diffusion modelling used for the flamelet table generation. For unity Lewis numbers, the transported and the adapted Bilger mixture fraction are almost identical. This does not hold true for nonunity Lewis numbers, and the effect on the adapted Bilger mixture fraction is shown in Appendix A.

\subsection{Species and temperature distributions}

Figure 3 shows radial profiles for the mean and the RMS temperature. As for the mixture fraction distributions, the most significant differences occur 
at $x / d=1$. Here, the mean profile is slightly shifted in a radial direction and the peak value is overestimated. The computed peak temperature fluctuation is lower than the measured one, and the fluctuations right above the pilot burner $(0.5 \leq r / d \leq 1.2)$ are not fully captured. This discrepancy is, at least partly, explained by the limited precision in the temperature measurement at this location and can therefore be considered as not significant. Additionally, the grid resolution close to the nozzle is not sufficient to resolve the shearlayer and the reaction zone. Overall, a good qualitative and quantitative agreement between the LES-FPV approach and the experiments is found.

In Figs. 4 and 5 , the mean and RMS profiles of species mole fractions of $\mathrm{CO}_{2}$ and $\mathrm{H}_{2} \mathrm{O}$ are shown. The development of the measured and the computed mean profiles again agree very well; only slight differences are found for the peak values. The RMS profiles of these two species exhibit features that are similar to the temperature profiles shown above. The computed fluctuations are underestimated at $x / d=1$ and the steep gradients around the thin flame zone at the downstream locations $x / d \leq 10$ are not sufficiently captured by the LES, due to the employed mesh resolution. Further comparisons of the mean and the RMS values for $\mathrm{CO}, \mathrm{H}_{2}, \mathrm{O}_{2}$, and $\mathrm{N}_{2}$ are provided in Appendix B, confirming that the LES captures the main flame characteristics.

Differences between the measured and the simulated results primarily occur in close proximity to the burner inlet plane (for $Z^{*}, T, X_{i}$ ). To analyze this further the flame structure in the mixture fraction space is presented in Fig.6. In this figure, the conditional mean values of the species mole fraction (DME, $\mathrm{H}_{2} \mathrm{O}, \mathrm{H}_{2}, \mathrm{CO}_{2}$ ) from the experiments are compared with instanta- 
neous and conditional mean data from the LES. To be consistent with the experimental data, the profiles of the DME mole fraction include important intermediate hydrocarbons $\left(\mathrm{CH}_{4}, \mathrm{C}_{2} \mathrm{H}_{4}, \mathrm{C}_{2} \mathrm{H}_{6}, \mathrm{CH}_{3}\right)$, which contribute to the Raman channel [26]. Again, differences between simulation and experiment are mainly found at $x / d=1$, while further downstream close agreement is observed. Except for $\mathrm{CO}_{2}$, the measured flame structure in close proximity to the nozzle is not well captured by the LES-FPV approach based on non-premixed flamelets. In the fuel-rich regions, especially, above the stoichiometric mixture fraction $\left(Z_{\mathrm{st}}^{*}=0.35\right)$, both the mole fractions of $\mathrm{H}_{2} \mathrm{O}$ and $\mathrm{H}_{2}$ and $\mathrm{DME}+\mathrm{C}_{x} \mathrm{H}_{y}$ consumption are significantly overestimated. Further downstream, the computed flame structure is in good agreement with the experimental data; the fuel rich-region, in particular, is better covered except for $\mathrm{H}_{2}$, which is always overestimated below $x / d=40$. Characteristic features such as the pyrolysis of DME and associated hydrocarbons under fuel-rich conditions $\left(Z^{*}>0.6\right.$, see Fig. 6) can also be observed in the underlying non-premixed FLUT (not shown here). This is one of the main differences in comparison with the corresponding $\mathrm{CH}_{4}$ flames and leads to greater robustness against local extinction due to the formation of reactive intermediates as described in [24].

At the farthest downstream location $(x / d=40)$ the measurements show the occurrence of conditional mean species data near $Z^{*}=1$. This feature is not captured by the LES-FPV, where the highest mixture fraction is around 0.9. In this particular range of mixture fraction $\left(0.9 \leq Z^{*} \leq 1.0\right)$ a significant reaction progress seems to be predicted by the experimental data. This difference can most likely be attributed to the underlying non-premixed flame 
structure and this can be seen from both the conditional mean and instantaneous simulation data. For non-unity Lewis numbers, the Bilger mixture fraction can become larger than one on the axis, which can be observed for non-premixed and premixed flamelet tables, see Fig 13 in Appendix A. This effect also increases the Bilger mixture fraction at the downstream position considered here. Similar to the discussion here (and in the appendix) for the species mole fraction, this difference for the mixture fraction at the farthest downstream position on the axis also shows up in the comparison of the effective Rayleigh cross-section, see Fig. 11 .

\section{3. $\mathrm{CH}_{2} \mathrm{O}$ and $\mathrm{OH}$-LIF signals}

Here we use the experimental $\mathrm{CH}_{2} \mathrm{O}$-LIF and OH-LIF signals for direct comparison with the simulation results. The procedure to calculate the LIF signals from the numerical data is described in Sections 2.2.1 and 2.2.2.

Figures 7 and 8 show the profiles of the normalized mean and RMS LIF signals for $\mathrm{OH}$ and $\mathrm{CH}_{2} \mathrm{O}$, respectively. The computed signals are generated using the method described in [23], where the LIF signals at each downstream location are normalized by the peak value of the profile at $x / d=5$. No significant differences between computed and measured mean OH-LIF signals can be observed up to $x / d=15$. Further downstream the computed profiles

become wider than the measured profiles. Furthermore, the peak values of the computed mean OH-LIF signals show a small radial shift towards the jet axis. The corresponding computed RMS profiles exhibit a similar structure, with the increased peak value and the radial shift of the profiles towards the axis.

For the mean $\mathrm{CH}_{2} \mathrm{O}$-LIF signals, the computed and measured radial pro- 
files are quite similar, although the measured profiles are slightly wider. The prediction of the radial positions and relative magnitudes of the peak $\mathrm{CH}_{2} \mathrm{O}$ LIF signals is excellent at all downstream locations except at $x / d=20$. From $x / d=10$ to $x / d=20$, significant differences for the mean profiles occur only close to the jet axis. The LES-FPV results show lower mean $\mathrm{CH}_{2} \mathrm{O}-\mathrm{LIF}$ signals than the experiments. As shown before in Fig. 2, this effect is related to the finding that the mixture fraction is between 0.9 and 1 at these particular axial locations. The underlying flame structure of a non-premixed flamelet is characterized by mixing in this particular range of mixture fraction and no significant reaction progress occurs. This could explain the underestimation of $\mathrm{CH}_{2} \mathrm{O}$ close to the jet axis. In the farthest downstream measurement plane $(x / d=25)$, where the maximum mixture fraction has further decreased, only small differences can be observed again. The profiles of the computed RMS $\mathrm{CH}_{2} \mathrm{O}$-LIF signals also agree well with experimental data, except that the experimental profiles are slightly broader, as is the case for the mean profiles of the $\mathrm{CH}_{2} \mathrm{O}$-LIF signals. Thus, except for the $\mathrm{CH}_{2} \mathrm{O}$-LIF signals in the vicinity of the centerline from $x / d=10$ to $x / d=20$, the overall agreement between measured and computed LIF signals, both for $\mathrm{CH}_{2} \mathrm{O}$ and $\mathrm{OH}$, is good.

Single-shot measurements in [23] showed that the $\mathrm{CH}_{2} \mathrm{O}$ and $\mathrm{OH}$ distributions exhibit distinct separation features. Coriton et al. [23] also showed that the gap between the occurrence of the two species increases in the downstream direction. Furthermore, LIF measurements showed that local overlapping of these species distributions is possible and the two species can be in close proximity. These features can also be identified in the flamelet ta- 
ble. Figure 9 shows the $\mathrm{CH}_{2} \mathrm{O}$ and $\mathrm{OH}$ distributions in the mixture fraction space for different scalar dissipations rates, $\chi_{\mathrm{st}}$. The minimum and maximum scalar dissipation rates are $\chi_{\mathrm{st}, \min }=0 \mathrm{~s}^{-1}$ and $\chi_{\mathrm{st}, \max }=1200 \mathrm{~s}^{-1}$, respectively. The minimum value corresponds to chemical equilibrium, and the maximum value corresponds to a flamelet solution near the quenching limit. This limit is evaluated during the flamelet look-up table generation using an iterative approach to determine a burning flamelet solution with a scalar dissipation rate that is as large as possible. At high scalar dissipation rates, an overlap between $\mathrm{CH}_{2} \mathrm{O}$ and $\mathrm{OH}$ can occur for $0.4 \leq Z \leq 0.55$. For lower values of $\chi_{\mathrm{st}}$, the formation and consumption of $\mathrm{CH}_{2} \mathrm{O}$ is restricted to the fuel-rich region. Since there is no $\mathrm{OH}$ in this particular region, the observed gap develops. This can be clearly observed in Fig. 10, where instantaneous distributions of $\mathrm{CH}_{2} \mathrm{O}$ and $\mathrm{OH}$ mole fractions are shown. Since the scalar dissipation rate decreases downstream, an increase in gap formation is observed, as prescribed by the underlying flamelet structure shown in Fig. 9. Close to the nozzle with higher scalar dissipation rates the $\mathrm{CH}_{2} \mathrm{O}$ and $\mathrm{OH}$ distributions are close and local overlapping can be observed. Existing gaps between the two species are small in comparison to those, which develop further downstream.

\subsection{Rayleigh signals/effective cross-sections}

Following the same approach as in the previous section, the Rayleigh signal from the LES is directly compared to the experimentally obtained value. As discussed in Section 2.2.3 the Rayleigh signal depends on the effective Rayleigh cross-section, which is a linear combination of the species mole fractions and the species specific cross-sections. Figure 11 shows the conditional mean values of the effective Rayleigh cross-sections obtained by 
means of experiment. The latter is based on the Rayleigh cross-section model for the hydrocarbon species introduced in [26]. The experimental values are compared to instantaneous and conditional mean results from the LES. Here, the computational results are obtained using the approach given by Eq. (9) without any model assumptions. Since a detailed reaction mechanism is used, the contributions from the hydrocarbon species can be considered directly.

At every axial location the computational results agree very well with experimental data in the fuel-lean regions. In these regions, very small amounts of hydrocarbons are found and the influence of the modelled hydrocarbon cross-section $\left(\sigma_{\text {Ray,HC }}\right)$ vanishes. This agreement for the Rayleigh crosssection corresponds to the good match of species profiles shown in Fig. 6 . In close proximity to $Z^{*}=1$, there is little decomposition of DME into intermediate hydrocarbons and the hydrocarbon cross-section is dominated by the cross-section of DME. In combination with the good agreement of DME mole fractions between the simulation and the experiment around $Z^{*}=1$ the effective Rayleigh cross-sections also agree well for all axial locations except for $x / d=40$. The differences at the farthest downstream position result from the differences in species distributions as shown in Fig. 6. A similar reasoning is likely to apply for the fuel-rich region at $x / d=1$, where the differences in conditional mean values of effective Rayleigh cross-sections can reach up to $30 \%$. The computed species distributions for DME and hydrocarbons also show significantly lower mole fractions than the experiment. For all other axial locations $(5 \leq x / d \leq 20)$ the LES-FPV results agree very well with the measurements and only small differences can be depicted. Since the variations occur in the range of significant DME decomposition they 
might be caused by both the differences in the species distributions and the assumptions of the Rayleigh cross-section model. To evaluate these model assumptions, both approaches presented in Section 2.2.3, are applied to the LES-FPV data. However, as shown in Appendix C, the variations based on the Rayleigh cross-section model are below $5 \%$ at almost every axial location.

\section{Conclusions}

In the present study, the flame structure of a piloted partially-premixed turbulent DME jet flame was investigated using an LES flamelet-progress variable approach. Recent experimental data, including velocity and multiscalar measurements using Raman, Rayleigh and LIF techniques, were used for validating and analyzing the computational results. In addition to the commonly applied comparison of standard quantities such as the temperature and species mole fractions, computations of measurement signals $\left(\mathrm{CH}_{2} \mathrm{O}\right.$ LIF, OH-LIF and Rayleigh scattering) have been included directly into the flamelet-progress variable approach.

Overall, flow field statistics between computations and measurements agree very well. Despite small differences in the direct proximity of the nozzle, no significant differences to the experimental data were found for the mean or the RMS profiles of the velocity and mixture fraction. The same trend could be observed for the temperature and major species. Analysis of the species distributions in mixture fraction space showed that the flame structure of the partially-premixed DME flame is captured well by the LESFPV approach based on non-premixed flamelets. Smaller differences in the flame structure were observed at the farthest downstream location. While 
experimental data showed significant reaction progress for mixture fraction values close to one, this is not fully captured in the LES-FPV computations, which might be partially attributed to the unity Lewis number approach.

Computed and measured LIF signals showed good agreement for $\mathrm{OH}$ throughout the observed flow field. Despite the generally good agreement for $\mathrm{CH}_{2} \mathrm{O}$-LIF profiles, some differences were observed in the vicinity of the jet centerline. The larger experimentally obtained signals suggest an increased reaction progress due to DME pyrolysis in the fuel rich region, which is not fully described by the underlying non-premixed flame structure. However, separation features of $\mathrm{CH}_{2} \mathrm{O}$ and $\mathrm{OH}$ concentration fields, experimentally observed using simultaneous $\mathrm{CH}_{2} \mathrm{O}$ - and $\mathrm{OH}-\mathrm{LIF}$, are qualitatively captured in the simulations. The gaps between the $\mathrm{CH}_{2} \mathrm{O}$ and $\mathrm{OH}$ distributions increase as the scalar dissipation rates decrease. This effect, which primarily occurs at further downstream locations, is fully captured by the underlying flamelet structure.

The experimentally obtained effective Rayleigh cross-sections, which are based on model assumptions for the very rich regions, agreed well with the computed cross-sections from the LES. It was shown that the model assumptions, although derived from laminar flame computations, are valid for the investigated turbulent DME flame in most of the flow field. The remaining differences for the effective Rayleigh cross-sections, especially very close to and at the farthest distance from the nozzle, result from the use of a single strain rate in the laminar flame calculations for the experimental post-processing. 


\section{Acknowledgements}

S. Popp, D. Messig, S. Hartl, F. Hunger and C. Hasse gratefully acknowledge the financial support by the Federal Ministry of Economics and Technology of Germany (project number 03ET7026B), by the Federal Ministry of Education and Research of Germany in the framework of Virtuhcon (project number 03Z2FN11) and by the Saxon Ministry of Science and Fine Arts and the European Union in the project BioRedKat (Project No. 100097882). Experiments performed at Sandia were supported by the U.S. Department of Energy, Office of Basic Energy Sciences, Division of Chemical Sciences, Geosciences, and Biosciences. Sandia National Laboratories is a multiprogram laboratory operated by Sandia Corporation, a Lockheed Martin Company, for the U.S. Department of Energy under contract DE-AC04-94-AL85000. The authors would like to thank Oliver Stein and Andreas Kronenburg for many fruitful discussions. 


\section{References}

\section{References}

[1] T. A. Semelsberger, R. L. Borup, H. L. Greene, Journal of Power Sources 156 (2006) $497-511$.

[2] T. Fleisch, A. Basu, R. Sills, Journal of Natural Gas Science and Engineering 9 (2012) $94-107$.

[3] International workshop on measurement and computation of turbulent flames, http://www.sandia.gov/TNF/abstract.html, 2014.

[4] R. Barlow, J. Frank, Symposium (International) on Combustion 27 (1998) 1087 - 1095.

[5] C. Schneider, A. Dreizler, J. Janicka, E. Hassel, Combustion and Flame 135 (2003) $185-190$.

[6] R. Barlow, A. Karpetis, Flow, Turbulence and Combustion 72 (2004) 427-448.

[7] R. Barlow, J. Frank, A. Karpetis, J.-Y. Chen, Combustion and Flame 143 (2005) $433-449$.

[8] R. Lindstedt, S. Louloudi, E. Váos, Proceedings of the Combustion Institute 28 (2000) $149-156$.

[9] Q. Tang, J. Xu, S. B. Pope, Proceedings of the Combustion Institute 28 (2000) $133-139$.

[10] J. Xu, S. B. Pope, Combustion and Flame 123 (2000) 281 - 307. 
[11] R. R. Cao, S. B. Pope, Combustion and Flame 143 (2005) 450 - 470.

[12] M. Roomina, R. Bilger, Combustion and Flame 125 (2001) 1176 - 1195.

[13] S. H. Kim, K. Y. Huh, Combustion and Flame 138 (2004) 336 - 352.

[14] S. Navarro-Martinez, A. Kronenburg, F. Di Mare, Flow, Turbulence and Combustion 75 (2005) 245-274.

[15] A. Kronenburg, M. Kostka, Combustion and Flame 143 (2005) $342-$ 356.

[16] H. Pitsch, H. Steiner, Proceedings of the Combustion Institute 28 (2000) $41-49$.

[17] H. Pitsch, H. Steiner, Physics of Fluids 12 (2000) 2541-2554.

[18] M. Ihme, H. Pitsch, Combustion and Flame 155 (2008) 90 - 107.

[19] M. Ihme, H. Pitsch, Physics of Fluids 20 (2008).

[20] A. Vreman, B. Albrecht, J. van Oijen, L. de Goey, R. Bastiaans, Combustion and Flame 153 (2008) $394-416$.

[21] K. N. Gabet, H. Shen, R. A. Patton, F. Fuest, J. A. Sutton, Proceedings of the Combustion Institute 34 (2013) 1447 - 1454.

[22] J. H. Frank, A. G. Hsu, J. Kuhl, in: Proceedings of the TNF 10.

[23] B. Coriton, M. Zendehdel, S. Ukai, A. Kronenburg, O. T. Stein, S.-K. Im, M. Gamba, J. H. Frank, Proceedings of the Combustion Institute 35 (2015) $1251-1258$. 
[24] F. Fuest, G. Magnotti, R. Barlow, J. Sutton, Proceedings of the Combustion Institute 35 (2015) 1235 - 1242.

[25] B. Connelly, B. Bennett, M. Smooke, M. Long, Proceedings of the Combustion Institute $32(2009) 879-886$.

[26] F. Fuest, R. S. Barlow, J.-Y. Chen, J.-Y. Dreizler, Combustion and Flame 159 (2012) 2533 - 2562.

[27] F. Nicoud, H. B. Toda, O. Cabrit, S. Bose, J. Lee, Physics of Fluids 23 (2011) 085106.

[28] C. D. Pierce, P. Moin, Journal of Fluid Mechanics 504 (2004) 73-97.

[29] R. Barlow, A. Karpetis, J. Frank, J.-Y. Chen, Combustion and Flame 127 (2001) $2102-2118$.

[30] W. L. Grosshandler, RADCAL: A Narrow-Band Model for Radiation Calculations in a Combustion Environment, Technical Report, NIST technical note 1402, 1993.

[31] N. Peters, Turbulent Combustion, Cambridge University Press, 2000.

[32] H. Pitsch, N. Peters, Combustion and Flame 114 (1998) 26 - 40.

[33] B. Fiorina, R. Baron, O. Gicquel, D. Thévenin, S. Carpentier, N. Darabiha, Combustion Theory and Modelling 7 (2003) 449-470.

[34] P. Domingo, L. Vervisch, D. Veynante, Combustion and Flame 152 (2008) $415-432$. 
[35] M. Tamura, P. A. Berg, J. E. Harrington, J. Luque, J. B. Jeffries, G. P. Smith, D. R. Crosley, Combustion and Flame 114 (1998) 502 - 514.

[36] E. W. Kaiser, T. J. Wallington, M. D. Hurley, J. Platz, H. J. Curran, W. J. Pitz, C. K. Westbrook, The Journal of Physical Chemistry A 104 (2000) 8194-8206.

[37] Z. Zhao, M. Chaos, A. Kazakov, F. L. Dryer, International Journal of Chemical Kinetics 40 (2008) 1-18.

[38] W. Ramaekers, J. van Oijen, L. de Goey, Flow, Turbulence and Combustion 84 (2010) 439-458. 


\section{Tables}

Table 1: Rayleigh cross sections

\begin{tabular}{cccccccc}
\hline$X_{i}$ & $\mathrm{CO}_{2}$ & $\mathrm{O}_{2}$ & $\mathrm{CO}$ & $\mathrm{N}_{2}$ & $\mathrm{CH}_{4}$ & $\mathrm{H}_{2} \mathrm{O}$ & $\mathrm{H}_{2}$ \\
\hline$\sigma_{\text {Ray }, i}$ & 2.39 & 0.86 & 1.24 & 1 & 2.13 & 0.69 & 0.21 \\
\hline \hline $\mathrm{OH}$ & $\mathrm{O}$ & $\mathrm{H}$ & $\mathrm{DME}$ & $\mathrm{C}_{2} \mathrm{H}_{2}$ & $\mathrm{C}_{2} \mathrm{H}_{4}$ & $\mathrm{C}_{2} \mathrm{H}_{6}$ & $\mathrm{CH}_{3}$ \\
\hline 1.49 & 0.17 & 0.15 & 9.28 & 4.01 & 5.80 & 6.30 & 1.58 \\
\hline
\end{tabular}

Table 2: Unburned gas mole fractions at $294 \mathrm{~K}$

\begin{tabular}{lcccccccc}
\hline & $\mathrm{DME}$ & $\mathrm{C}_{2} \mathrm{H}_{2}$ & $\mathrm{H}_{2}$ & $\mathrm{~N}_{2}$ & $\mathrm{O}_{2}$ & $\mathrm{Ar}$ & $\mathrm{H}_{2} \mathrm{O}$ & $\mathrm{CO}_{2}$ \\
\hline Jet & 0.2 & - & - & 0.6237 & 0.1673 & 0.0075 & 0.0008 & 0.0003 \\
Pilot & - & 0.0218 & 0.0878 & 0.6793 & 0.1718 & 0.0077 & 0.0012 & 0.0303 \\
Co-flow & - & - & - & 0.7746 & 0.2078 & 0.0093 & 0.008 & 0.0004 \\
\hline
\end{tabular}




\section{Figures}
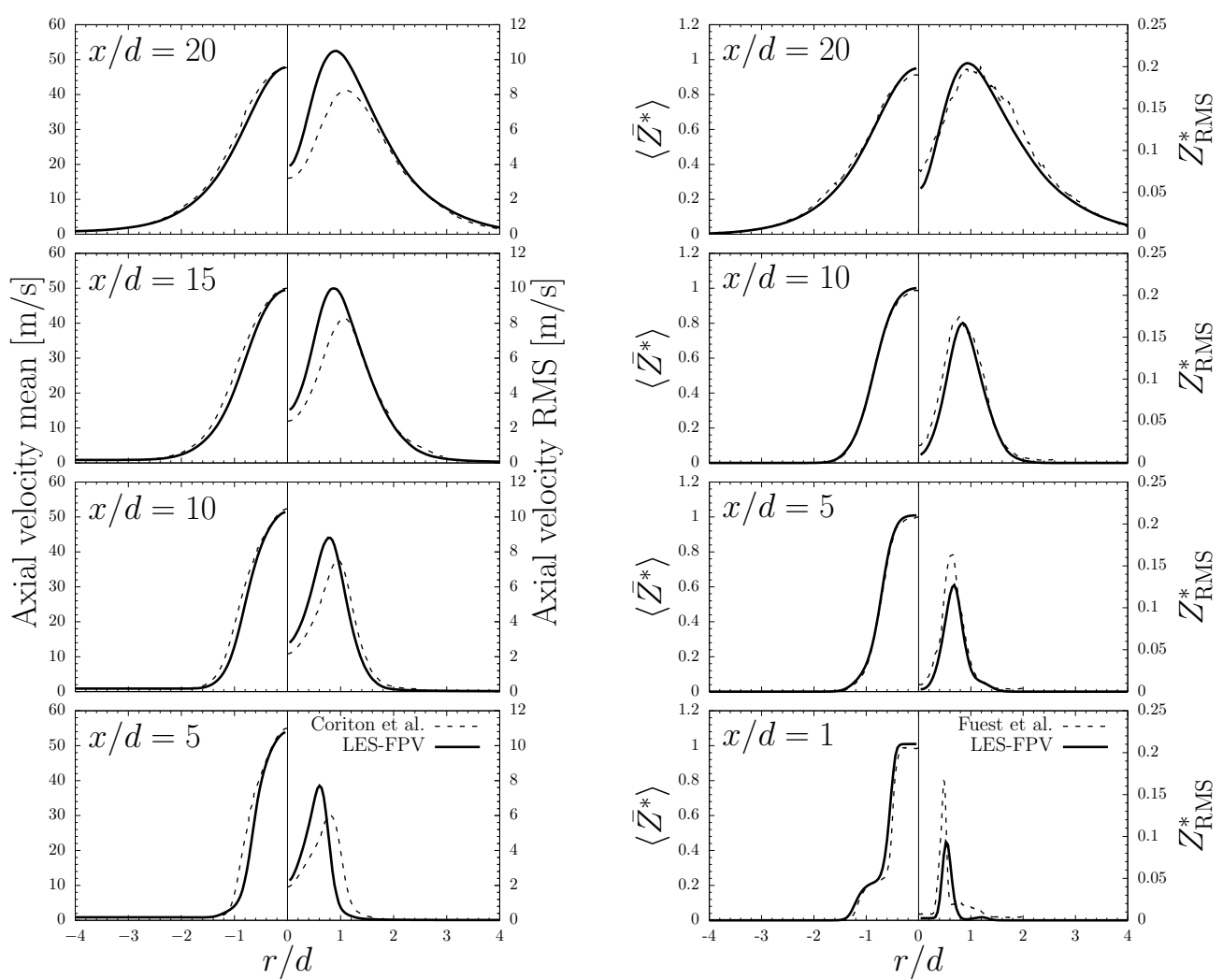

Figure 1: Mean and RMS of axial velocity

Figure 2: Comparison of the experimental at positions $x / d=5,10,15$ and 20, LES preand computed radial profiles of the mean dictions and experimental data from 23 are shown.

and the RMS adapted Bilger mixture fraction at the axial positions $x / d=1,5,10$ and 20 , respectively. 


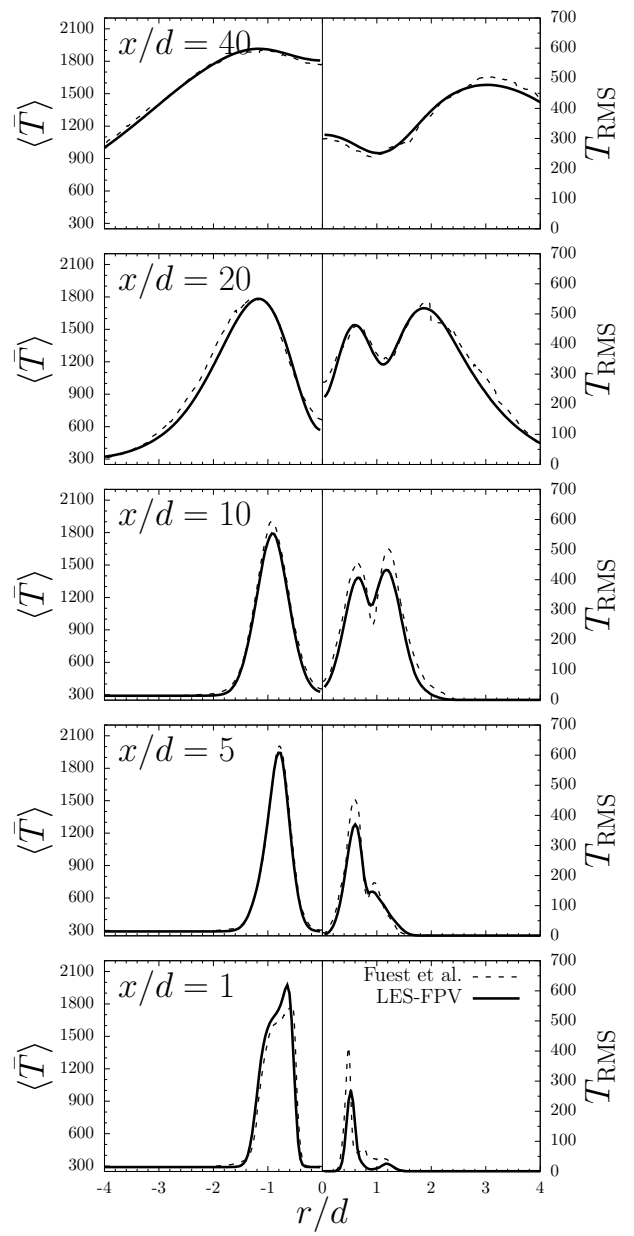

Figure 3: Comparison of the experimental and computed radial profiles of the mean and the RMS temperature at the axial positions $x / d=1,5,10,20$ and 40 , respectively. 

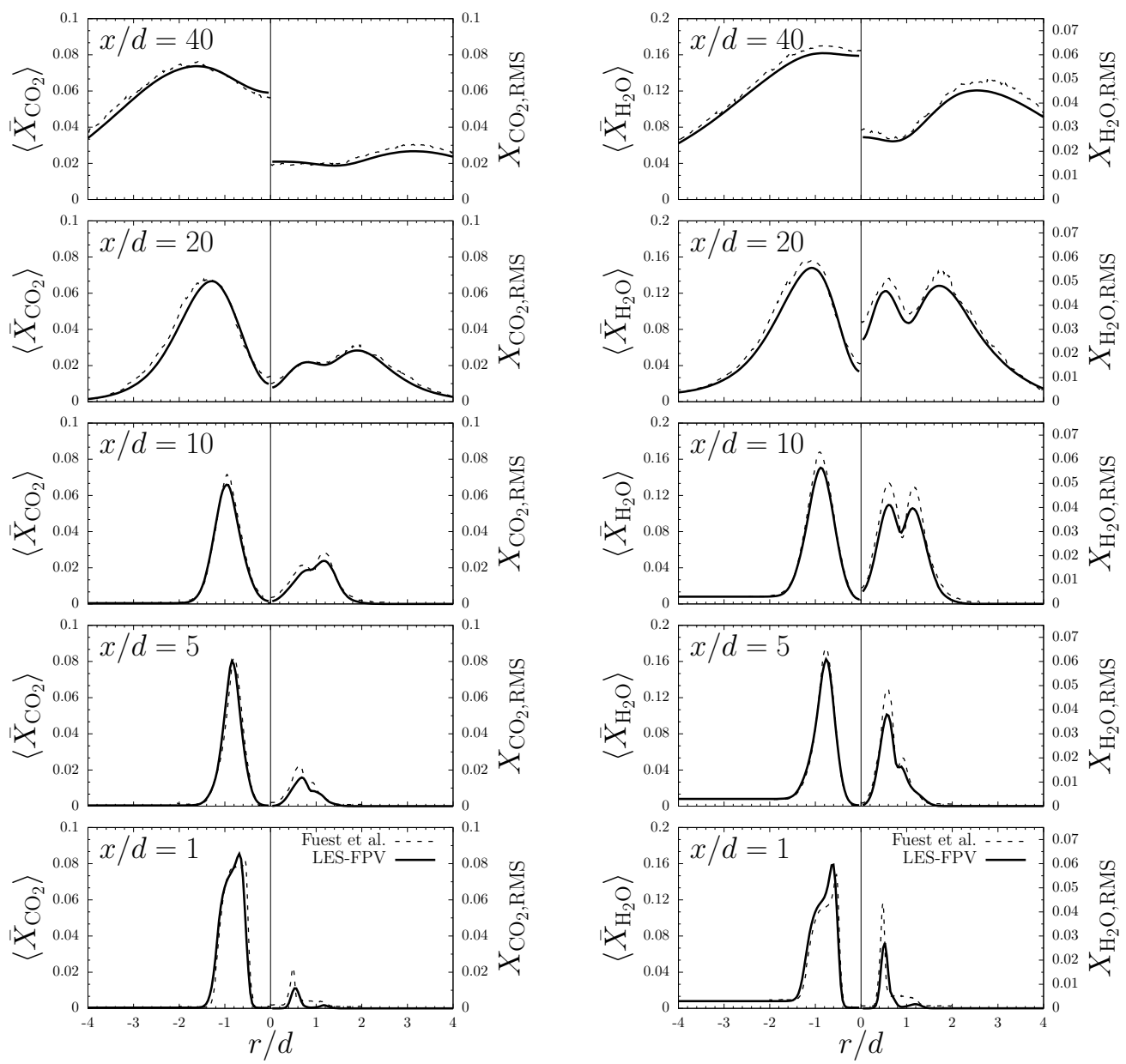

Figure 4: Comparison of the experimental Figure 5: Comparison of the experimental and computed radial profiles of the mean and computed radial profiles of the mean and the RMS $\mathrm{CO}_{2}$ mole fractions, using non- and the RMS $\mathrm{H}_{2} \mathrm{O}$ mole fractions, using nonpremixed flamelets with unity Lewis num- premixed flamelets with unity Lewis numbers, at the axial positions $x / d=1,5,10,20$ bers, at the axial positions $x / d=1,5,10,20$ and 40 , respectively. and 40 , respectively. 

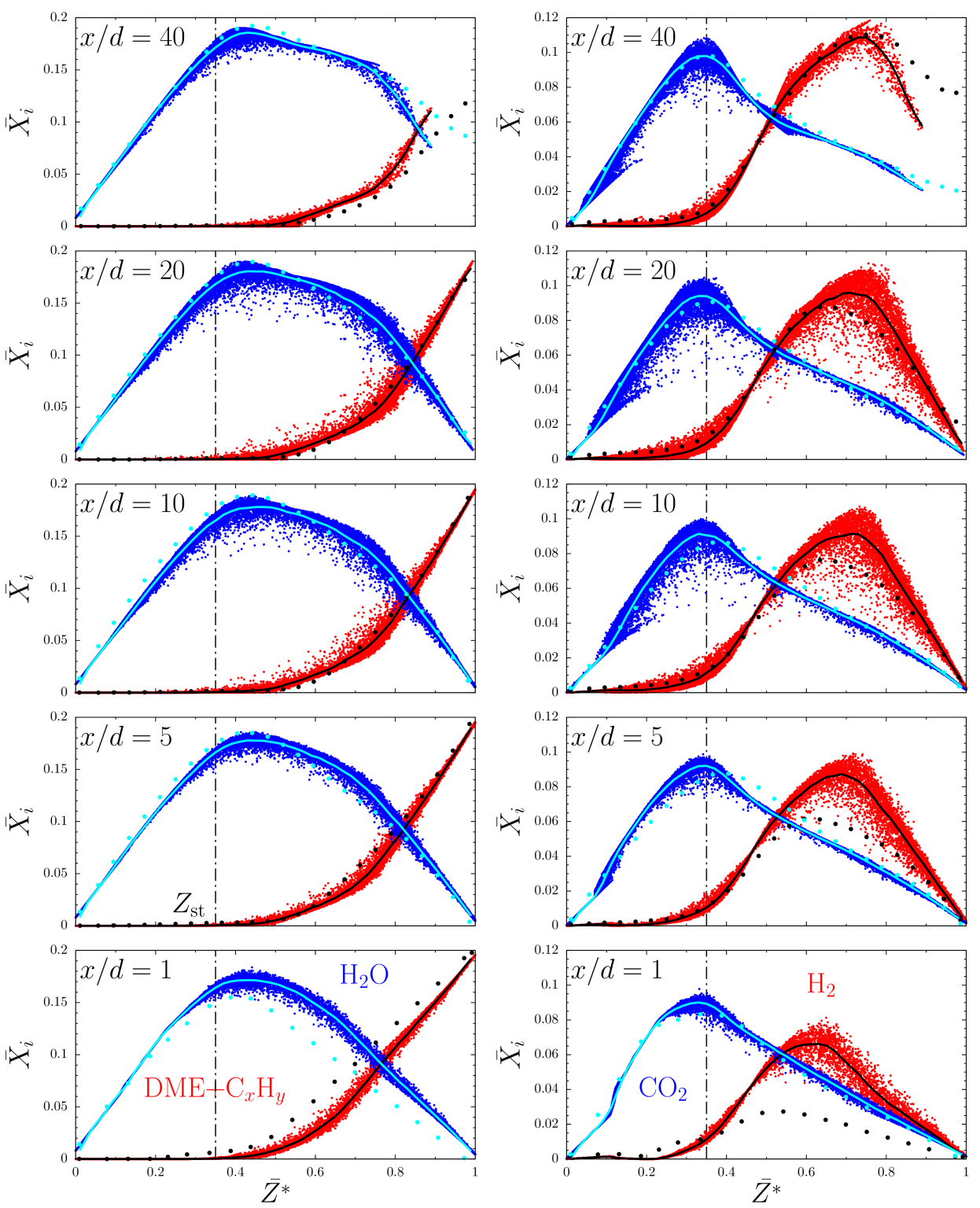

Figure 6: Comparison of the experimental and numerical conditional mean species distributions and instantaneous LES data at the axial positions $x / d=1,5,10,20$ and 40, respectively. The large dots represent the conditional mean values from the experiment and the lines represent the conditional mean values from the LES. The instantaneous LES data is represented by the point clouds. The dashed lines denote the stoichiometric mixture fraction. 

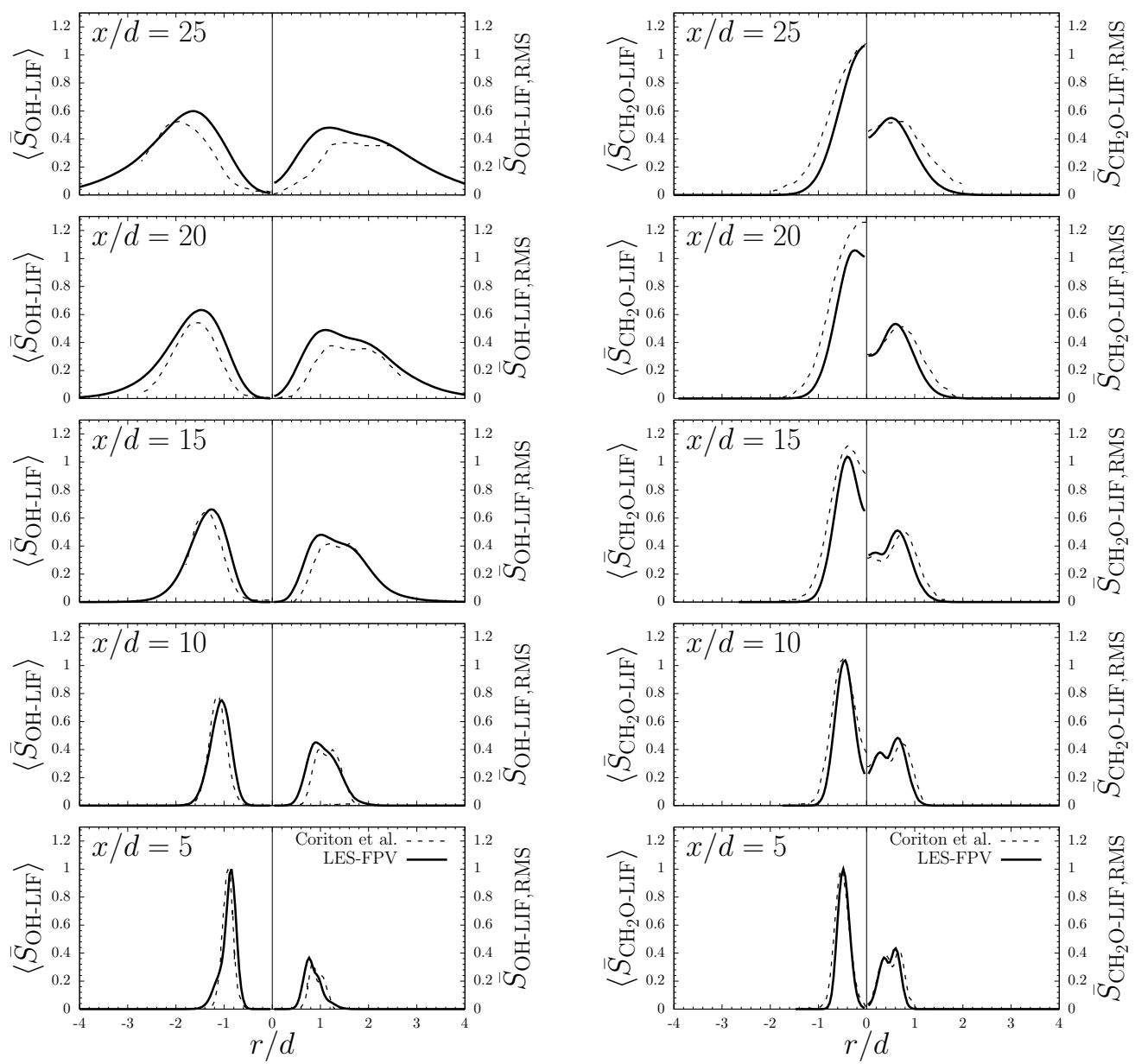

Figure 7: Comparison of experimental and Figure 8: Comparison of experimental computed mean and RMS LIF signals for and computed mean and RMS LIF signals $\mathrm{OH}$ at the axial positions $x / d=5,10,15,20$ for $\mathrm{CH}_{2} \mathrm{O}$ at the axial positions $x / d=$ and 25 , respectively. $5,10,15,20$ and 25 , respectively. 

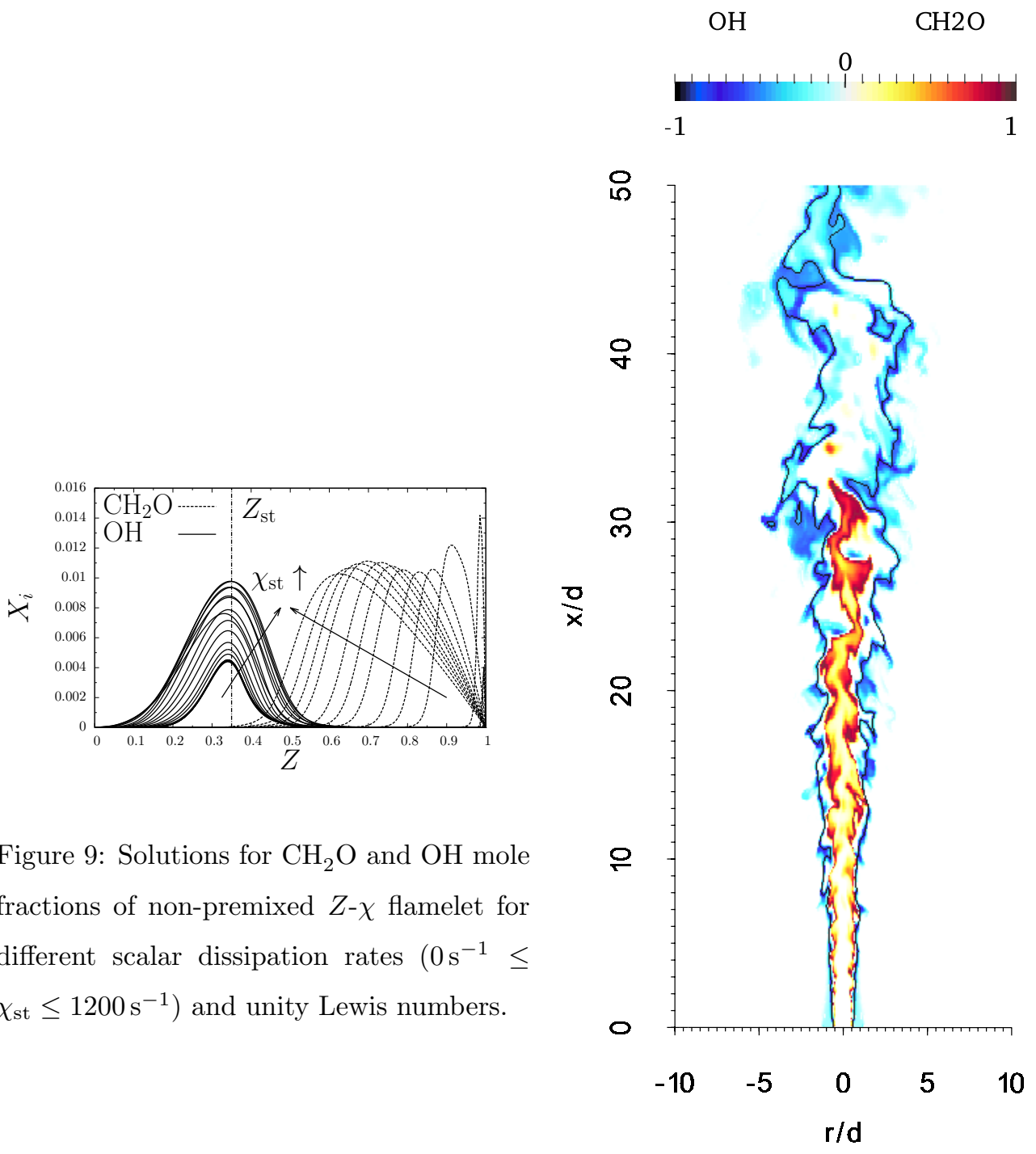

Figure 9: Solutions for $\mathrm{CH}_{2} \mathrm{O}$ and $\mathrm{OH}$ mole fractions of non-premixed $Z$ - $\chi$ flamelet for different scalar dissipation rates $\left(0 \mathrm{~s}^{-1} \leq\right.$ $\left.\chi_{\mathrm{st}} \leq 1200 \mathrm{~s}^{-1}\right)$ and unity Lewis numbers.

Figure 10: LES snapshot of the instantaneous and normalized $\mathrm{CH}_{2} \mathrm{O}$ and $\mathrm{OH}$ mole fractions on a refined mesh $(400 \times 160 \times 160$ cells), showing the gap between the species distributions. The black line denotes the stoichiometric mixture fraction contour. 


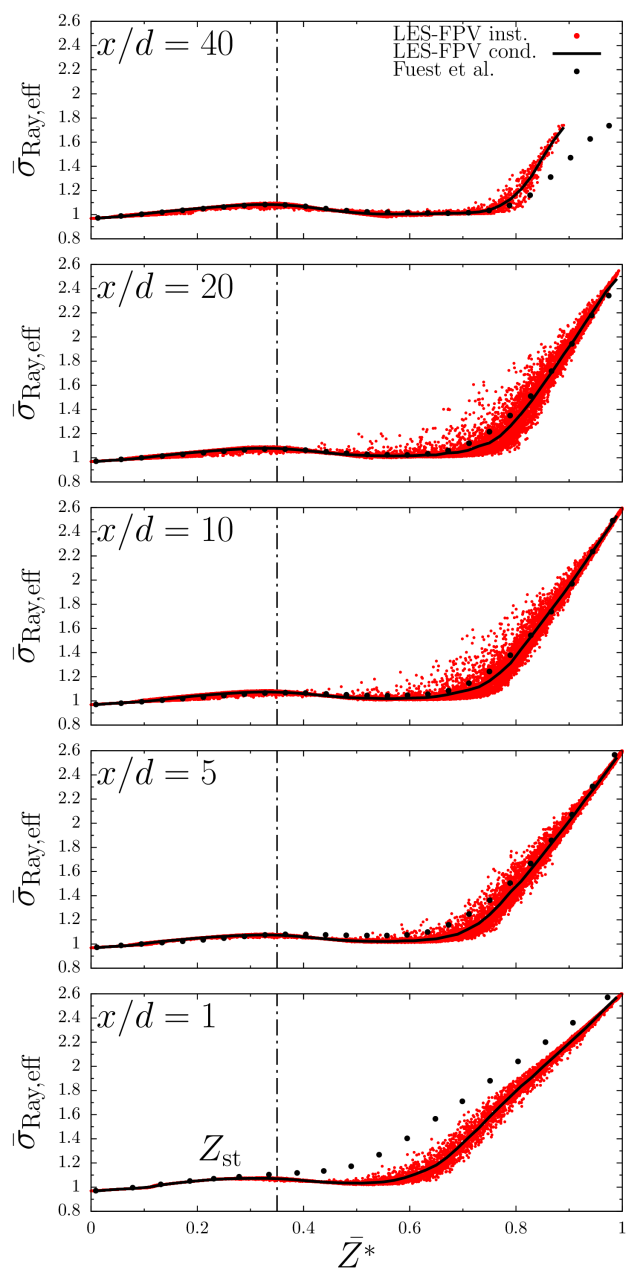

Figure 11: Comparison of the experimental and computed conditional mean effective Rayleigh cross-sections as well as instantaneous LES data at the axial positions $x / d=$ 1, 5, 10, 20 and 40, respectively. The large black dots represent the conditional mean values from the experiment and the black lines represent the conditional mean values from the LES. The instantaneous LES data is represented by the red point clouds. 

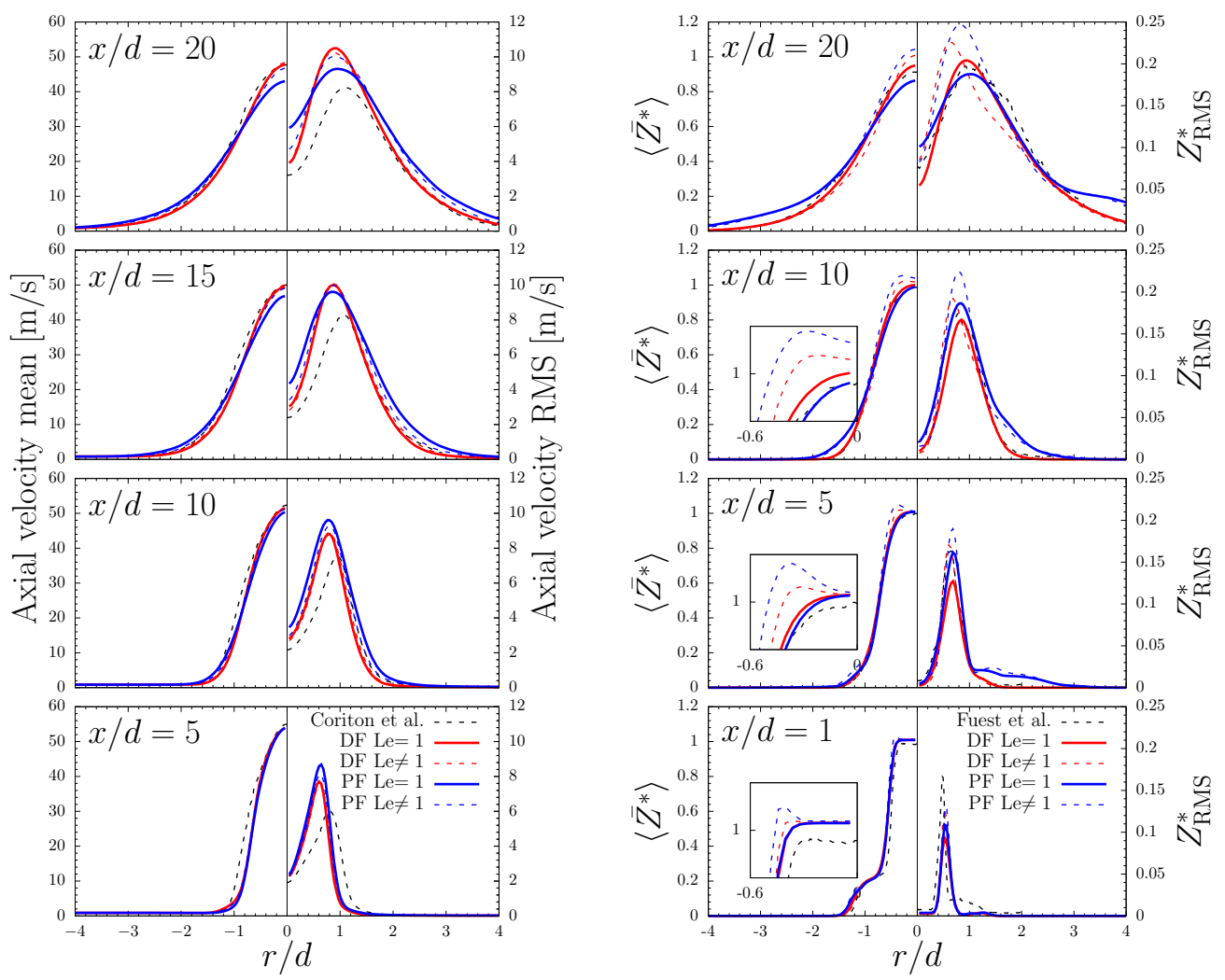

Figure 12: Mean and RMS of axial velocity at positions $x / d=5,10,15$ and 20, LES preFigure 13: Comparison of the experimental and computed radial profiles of the mean dictions based on premixed (PF) and nonand the RMS adapted Bilger mixture fracpremixed (DF) flamelets with either unity tion at the axial positions $x / d=5,10,15$ Lewis number or non-unity Lewis numbers and 20, respectively. The LES results are and experimental data from [23] are shown. based on premixed (PF) and non-premixed (DF) flamelets with either unity Lewis number or non-unity Lewis numbers. 


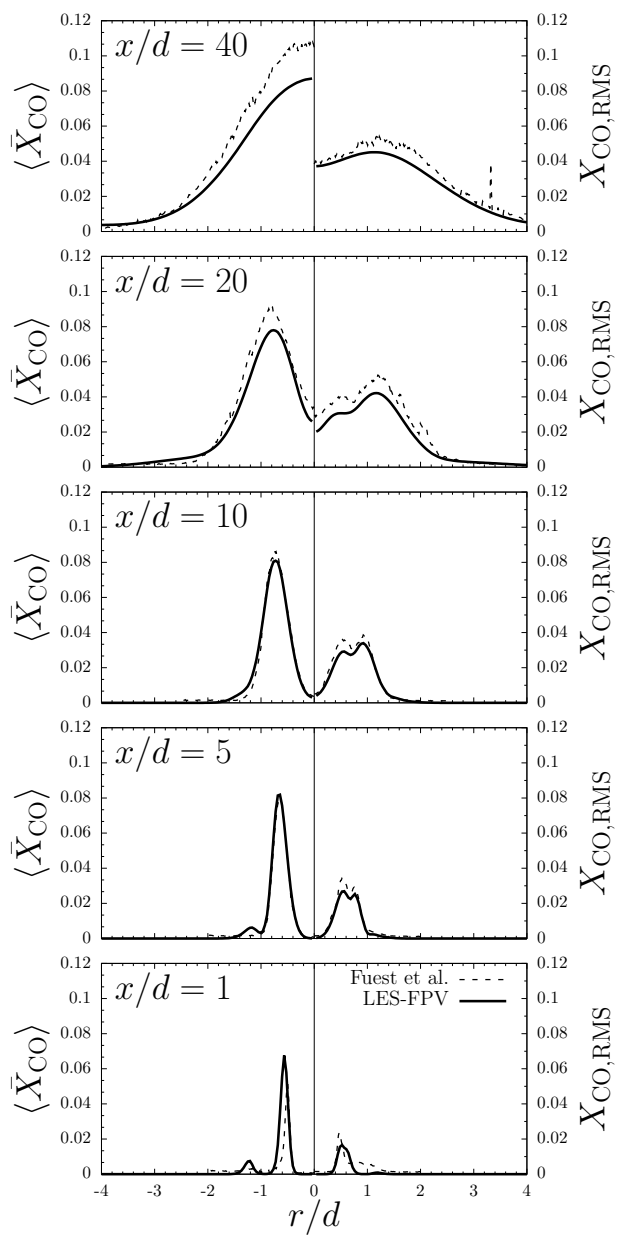

Figure 14: Comparison of the experimental and computed radial profiles of the mean and the RMS CO mole fractions, using nonpremixed flamelets with unity Lewis numbers, at the axial positions $x / d=1,5,10,20$ and 40 , respectively.
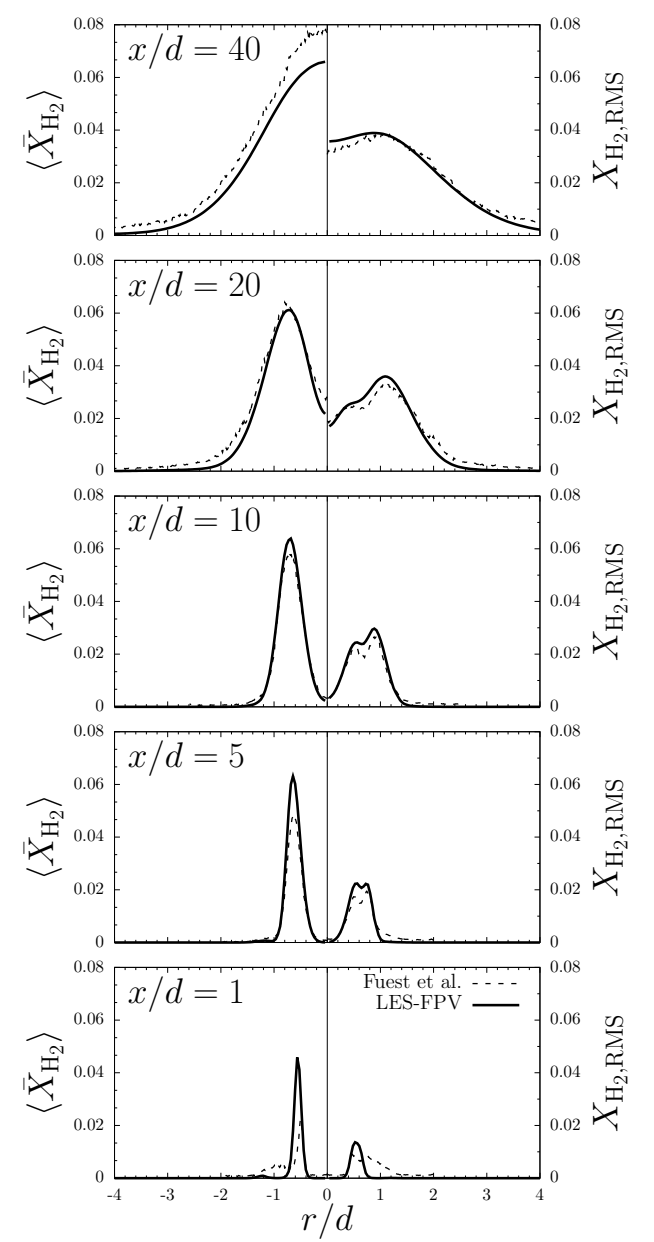

Figure 15: Comparison of the experimental and computed radial profiles of the mean and the RMS $\mathrm{H}_{2}$ mole fractions, using nonpremixed flamelets with unity Lewis numbers, at the axial positions $x / d=1,5,10,20$ and 40 , respectively. 

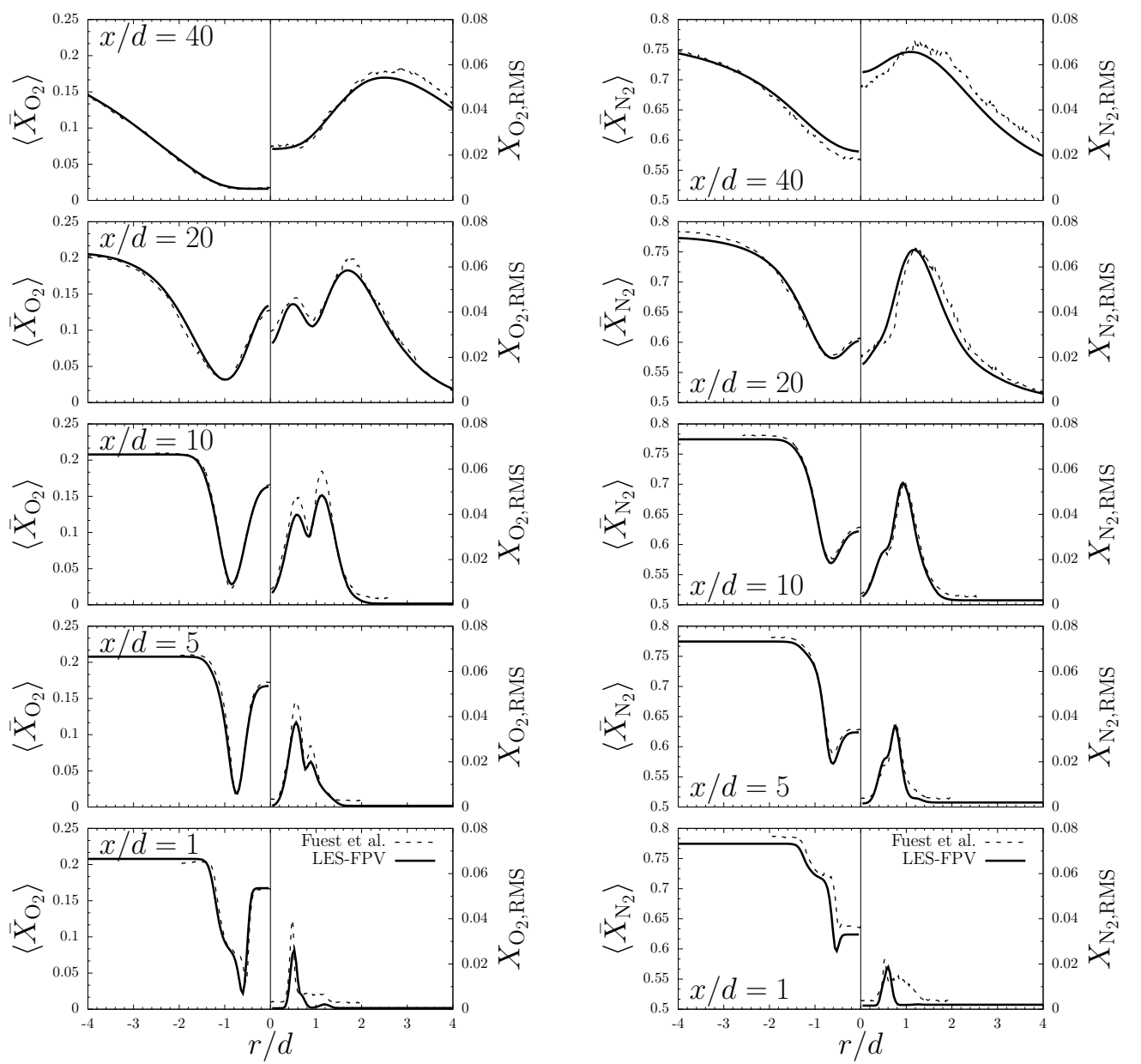

Figure 16: Comparison of the experimental and computed radial profiles of the mean and the RMS $\mathrm{O}_{2}$ mole fractions, using nonpremixed flamelets with unity Lewis numbers, at the axial positions $x / d=1,5,10,20$ and 40 , respectively.

Figure 17: Comparison of the experimental and computed radial profiles of the mean and the RMS $\mathrm{N}_{2}$ mole fractions, using nonpremixed flamelets with unity Lewis numbers, at the axial positions $x / d=1,5,10,20$ and 40 , respectively. 

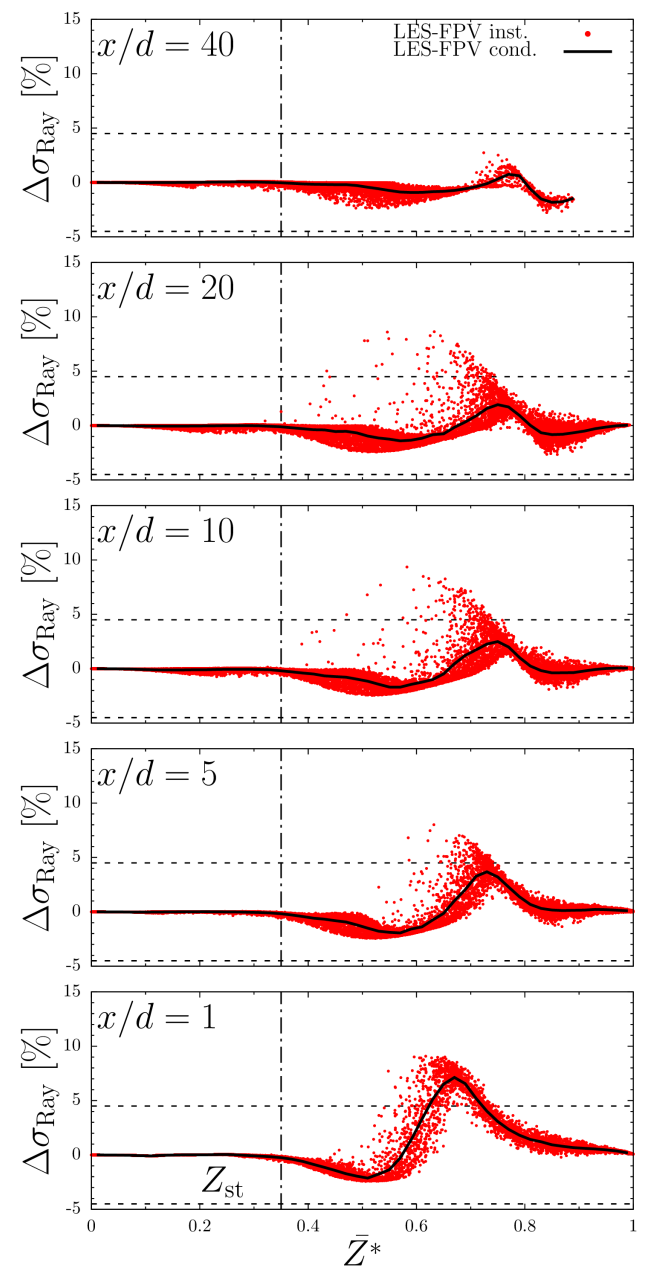

Figure 18: Instantaneous samples (points) and conditional mean values (lines) of the deviation in percent between effective Rayleigh cross-sections with and without the hydrocarbon model obtained by the LES at the axial positions $x / d=1,5,10,20$ and 40 , respectively. The dashed lines denote the stoichiometric mixture fraction. The estimated error bands of $\pm 4.5 \%$ by Fuest et al. [26] are indicated. 

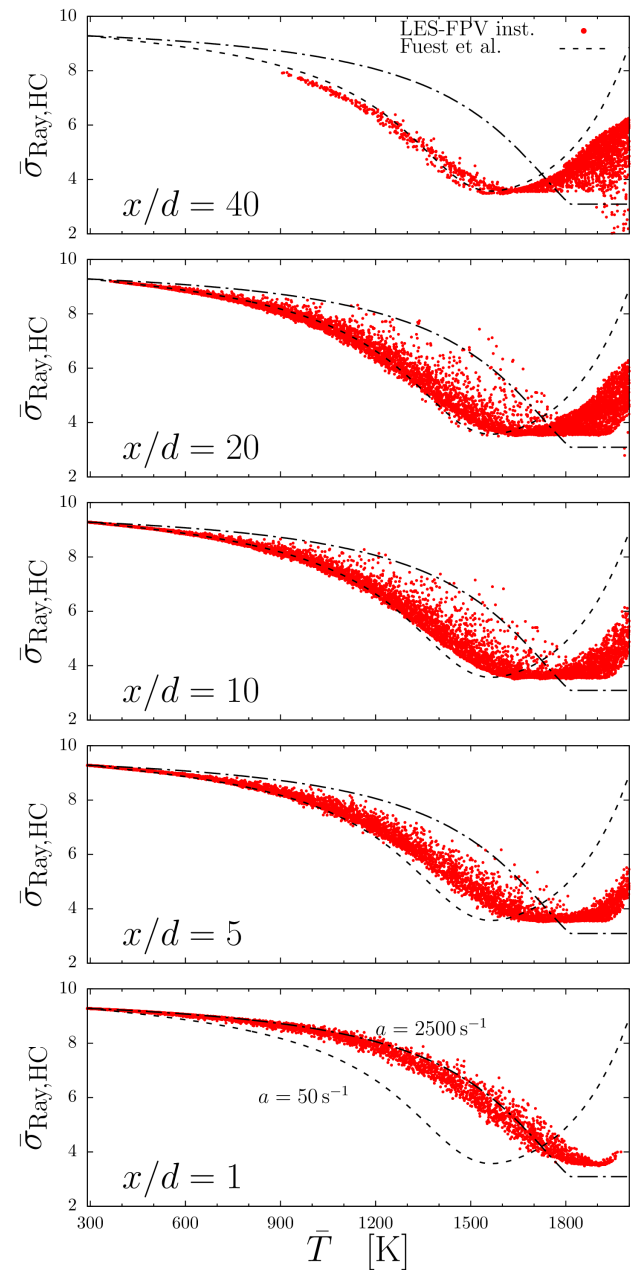

Figure 19: Instantaneous samples (points) of the Rayleigh cross-section model $\sigma_{\text {Ray,HC }}$ obtained by the LES and from 1D laminar flame calculation for two different strain rates $a=50 \mathrm{~s}^{-1}$ and $a=2500 \mathrm{~s}^{-1}$ at the axial positions $x / d=1,5,10,20$ and 40 , respectively. 


\section{Appendix A. Comparison of different flame structures for tabula- tion}

The results presented in the main section use tabulated non-premixed flamelets. As shown by Vreman et al. 20] for the Sandia flame D and F, premixed flamelets (PFs) can be more suitable for these particular flames compared to non-premixed flamelets (DFs) using a unity Lewis number approach. Based on experimental investigations, Gabet et al. 21] concluded that DME pyrolysis leads to increased amounts of $\mathrm{H}_{2}$ in the fuel-rich region. This does not occur for $\mathrm{CH}_{4}$ flames, which suggests, especially for the rich part, that non-unity Lewis numbers should be investigated. Thus, in addition, two flamelet tables (PF and $\mathrm{DF}$ ) with non-unity Lewis numbers were generated. Figure 12 shows radial profiles of the mean and the RMS velocity for the different look-up tables. Both diffusion modelling approaches provide identical velocity statistics with a FLUT based on a non-premixed flamelet, and these results agree well with the SPIV measurement. However, results obtained using premixed flamelets show significant differences. At axial locations $x / d \leq 15$ a premixed FLUT with Le $\neq 1$ yields comparable results to the non-premixed FLUT. However, with increasing axial distance the jet breakup is slightly overestimated. By contrast, the results from the premixed FLUT Le $=1$ exhibits significant differences to the experimental data and also to the results of the other look-up tables. An early jet breakup with a significantly reduced centerline velocity can be observed using this FLUT, especially at locations far downstream.

The deviations between the four look-up tables can also be depicted for the mixture fraction. Figure 13 shows the mean and the RMS profiles of the 
adapted Bilger mixture fraction as proposed in [26]. One major characteristic is that a non-unity Lewis number approach leads to a notable overshoot of the profiles near the centerline, which is not seen using unity Lewis numbers. In fact, preferential diffusion leads to mixture fraction values $Z^{*}$ larger than 1 , an effect which is not possible for unity Lewis numbers. Above $x / d=10$ this phenomena can no longer be observed. However, the results still show significant deviations from the experimental data. At the farthest downstream measurement plane, the mixture fraction is considerably reduced in the core area of the jet. The previously mentioned early jet breakup of the test case with a premixed FLUT and unity Lewis number approach also affects the mixture fraction field. While the results agree well with the corresponding non-premixed FLUT below $x / d=10$, the mixture fraction is drastically reduced near the jet axis with increasing axial distance from the nozzle. Based on the results, a non-premixed flamelet was chosen using unity Lewis number approach. A comparison of the mixture fraction fields, especially, indicates that preferential diffusion effects are not dominant in this flame, not even in the close proximity of the nozzle.

\section{Appendix B. Detailed comparison of radial species profiles}

Section 4.2 presents spatially and temporally averaged radial profiles of selected species. This appendix provides the remaining comparisons of all species, both for the mean and the RMS values, obtained from Raman measurements in Figs 14 17. 


\section{Appendix C. Evaluation of the Rayleigh cross-section model for intermediate hydrocarbons}

The Rayleigh cross-section model proposed in [26] is based on laminar flame calculations, with variable strain rates. For the computation of the temperature-dependent hydrocarbon cross-section, one particular strain rate was chosen to represent the entire flame. In turbulent flames, strain can exhibit strong variations. In order to estimate the error which occurs from the application of the Rayleigh cross-section model and from neglecting the effect of varying strain rates, species mole fractions obtained from the LESFPV computations were used to compare the model-based approach given by Eq. (10) to the computations without any assumptions given by Eq. (9). The same temperature-dependence of $\sigma_{\text {Ray,HC }}$ that was used to process the experimental data from 1D Raman/Rayleigh measurements presented in [24] was used in this evaluation. The resulting error between the effective Rayleigh cross-sections is computed by

$$
\Delta \sigma_{\text {Ray }}=\frac{\sigma_{\text {Ray,eff,ref }}-\sigma_{\text {eff,exp }}}{\sigma_{\text {Ray,eff,ref }}} \cdot 100 \%
$$

where $\sigma_{\text {Ray,eff,ref }}$ is the effective Rayleigh cross-section without any model assumptions and $\sigma_{\text {Ray,eff,exp }}$ is computed with the modelling approach, respectively. Figure 18 shows the instantaneous errors in the mixture fraction space as well as the conditional mean values of this distribution at different axial locations. At all axial locations, the error for stoichiometric and leaner mixtures is negligibly small. For larger mixture fractions, the impact of the cross-section model increases. Due to varying strain rates along the flame the computed error of the cross-section model also varies with axial distance 
from the nozzle. In close proximity to the nozzle, at $x / d=1$, the conditional mean error reaches up to $7 \%$, while individual samples exhibit errors of around $10 \%$. However, in this case the Rayleigh-cross section model is based on a 1D flame calculation with a comparatively low strain rate and strain is very high near the nozzle. With increasing axial distance and decreasing strain rates, the computed error becomes smaller and the conditional mean error is below $5 \%$. These observations agree with the estimation in [26], where an error of $4.5 \%$ was determined from 1D laminar flame calculations with varying strain rates. The conditional mean error could be reduced by using models based on 1D laminar flame calculations with higher strain rates for the lower axial locations. This is shown in Fig. 19 which compares the Rayleigh cross-section model obtained by the LES and two models from 1D laminar flame calculations. The model with the low strain rate $\left(a=50 \mathrm{~s}^{-1}\right)$, which was also applied for the experimental data, shows significant differences compared to the LES data at the lowest axial location. In this region the model with the increased strain rate $\left(a=2500 \mathrm{~s}^{-1}\right)$ is more appropriate and shows much better agreement with the computational results. However, the application of the model will still be limited and it will not correct for outliers, in particular those found towards higher axial locations. 